\title{
Insulator-quantum Hall transition in antidot lattices
}

\author{
Seiji Uryu* and Tsuneya Ando \\ Institute for Solid State Physics, University of Tokyo \\ 5-1-5 Kashiwanoha, Kashiwa-shi, Chiba, Japan
}

\begin{abstract}
Localization in antidot lattices subjected to a magnetic field is numerically studied. Oscillations of the localization length with periods of $\Phi_{0} / 2$ and $\Phi_{0}$, where $\Phi_{0}$ is a flux quantum $h / e$, as a function of flux passing through a unit cell are obtained and an insulator-quantum Hall transition point where the Fermi level crosses extended states is identified. It is revealed that extended states float up in energy and disappear at a critical magnetic field. The results are in good agreement with recent experiments.
\end{abstract}

\section{INTRODUCTION}

An antidot lattice is a two-dimensional (2D) electron system modulated by an array of strong repulsive potential called antidot. This system is usually realized in semiconductor heterostructures such as $\mathrm{GaAs} / \mathrm{Al}_{x} \mathrm{Ga}_{1-x} \mathrm{As}$ with the use of microfabrication technique. In recent experiments on insulator-quantum Hall transition in antidot lattices, ${ }^{1}$ characteristic and intriguing localization phenomena were observed. The purpose of this paper is to clarify localization in antidot lattices in the presence of magnetic fields.

In actual antidot lattices in magnetic fields, electrons at the Fermi energy are localized due to inherent disorder except at critical magnetic fields of insulator-quantum Hall transition and plateau-plateau transition of the quantum Hall effect. The main cause of disorder is not impurities but fluctuations of the antidot potential itself. ${ }^{2,3}$ The experimental results show the peculiar localization phenomena such as an oscillation of resistivity as a function of flux passing through a unit cell. The period is $\Phi_{0} / 2$ near the vanishing field and $\Phi_{0}$ around the field corresponding to an insulator-quantum Hall transition point or higher, where $\Phi_{0}$ is the flux quantum $h / e$.

The former is similar to the Al'tshuler-Aronov-Spivak oscillation $^{4}$ in metallic cylinders in a weak localization regime, but actually quite different because it is observed in a strong localization regime and involves the oscillation of the localization length itself. This has been confirmed by numerical calculations based on a Thoulessnumber method. ${ }^{5}$ The latter is similar to the AharonovBohm (AB) type oscillation, ${ }^{6,7}$ which is an oscillation of resistivity appearing around a magnetic field given by a commensurability condition of $2 R_{c}=a$ with $R_{c}$ being the classical cyclotron radius and arises from periodic orbits encircling an antidot. ${ }^{7-10}$

The insulator-quantum Hall transition in homogeneous 2D systems has been intentionally studied concerning the behavior of extended states under mixing of the Landau levels. In strong magnetic fields states at the center of broadened Landau levels are extended, ${ }^{11-13}$ while in a zero magnetic field all states are localized according to the scaling theory of localization. ${ }^{14}$ A floating picture was proposed where all the extended states float up towards the infinitely large energy in a zero field limit ${ }^{15,16}$ and it was extended into a global phase diagram $^{17}$ including the fractional quantum Hall effect. Various experimental ${ }^{18-20}$ and theoretical ${ }^{21-26}$ studies have been performed on this subject.

Some recent studies based on a tight-binding model with short range random potentials, ${ }^{27,28}$ showed that extended states with positive Chern number ${ }^{29,30}$ merge another extended states with negative Chern number and disappear together, leading to the disappearance of all the extended states in the zero field limit. Some of latest studies have reported floating up of extended states in tight-binding models with disorder potentials of nonzero correlation length. ${ }^{31,32}$ The antidot lattice constitutes an intriguing prototype system for the study of such localization phenomena.

The purpose of this paper is to elucidate the localization effect in antidot lattices in magnetic fields. This paper is organized as follows. In Sec. II, the model and method are described. In Sec. III, numerical results are given and discussed. Finally, a summary is given in Sec. IV.

\section{MODEL AND METHOD}

\section{A. Antidot lattice}

We consider a 2D array of quantum-wire junctions as a model of antidot lattice. ${ }^{5,9}$ Figure 1 (a) shows a model of a hexagonal antidot lattice where a system consists of quantum-wire junctions with an antidot labeled by 1 in the center and four antidots labeled by 2 to 5 , constrictions between which are considered as leads. In this model transport in an antidot lattice is represented by a scattering ( $\mathrm{S}$ ) matrix given by combinations of $\mathrm{S}$ matrices describing scattering in each quantum-wire junction. Figure 1(b) shows a single quantum-wire junction used in calculations of $\mathrm{S}$ matrices. We define an $\mathrm{S}$ matrix for not only traveling modes but also for evanescent modes because the constriction between neighboring junction$\mathrm{s}$ is not long enough for all evanescent modes to decay completely.

We use the potential of an antidot $v(\mathbf{r})$ in the WignerSeitz cell at the origin given by

$v(\mathbf{r})=U_{0}\left|\cos \left(\frac{\pi \mathbf{a}_{1} \cdot \mathbf{r}}{a^{2}}\right) \cos \left(\frac{\pi \mathbf{a}_{2} \cdot \mathbf{r}}{a^{2}}\right) \cos \left[\frac{\pi\left(\mathbf{a}_{1}-\mathbf{a}_{2}\right) \cdot \mathbf{r}}{a^{2}}\right]\right|^{4 \beta / 3}$,

with $U_{0}$ being a maximum of the potential, $a$ a lattice constant, $\beta$ a parameter describing steepness of the potential, $\mathbf{a}_{1}=(\sqrt{3} a / 2, a / 2)$, and $\mathbf{a}_{2}=(0, a){ }^{2,3,5}$ Then, the 
potential $V(\mathbf{r})$ of the antidot lattice is represented by

$$
V(\mathbf{r})=\sum_{i} v\left(\mathbf{r}-\mathbf{R}_{i}\right),
$$

where $\mathbf{R}_{i}$ is the $i$ th lattice point. We define antidot diameter $d$ from a potential profile of Eq. (2.1) at the Fermi energy along the directions of $\mathbf{a}_{1}$ ( or $\mathbf{a}_{2}$ or $\mathbf{a}_{1}-\mathbf{a}_{2}$ ). We set the upper limit of antidot potential as $1.5 \times E_{F}$, which is reasonable for the actual system with antidots realized by shallow holes on the surface of samples. ${ }^{1}$

Two kinds of disorders are taken into account. One is impurity potential and the other a disorder in the antidot potential. The former is characterized by the mean free path $l_{e}$ in the absence of the antidot potential. The latter may arise from various fluctuations of each antidot potential such as its diameter, center position, shape, boundary roughness, and so on. Among these fluctuations, that of the center position is not considered to be large in typical systems with lattice constant $\sim 100$ nm. The boundary roughness will be smeared out to large extent and not important because of the presence of depletion layers. Effects of fluctuations of the shape can be approximately included by fluctuations of the antidot diameter. Therefore, we consider fluctuations of the antidot diameter only as the representative source of the disorder. ${ }^{2,3}$ The amount of the fluctuation is characterized by $d_{f}=\sqrt{\left\langle d^{2}\right\rangle-\langle d\rangle^{2}}$, where $\langle\cdots\rangle$ represents the average over different antidots.

The fluctuation of the antidot diameter is introduced as follows. At first, a set of $\mathrm{S}$ matrices for quantumwire junctions with different values of the diameter of the centered antidot and those at the corners are calculated. Then, an S matrix for a whole antidot lattice is obtained by connecting S matrices for junctions with antidot diameters determined at random but in such a way that there is no discontinuous change in the potential profile.

We calculate an S matrix numerically for a quantumwire junction on a square lattice described by a nearestneighbor tight-binding model. The lattice constant $a^{\prime}$ of the lattice is chosen to be $a / a^{\prime}=7$, for which the system can simulate well an actual $2 \mathrm{D}$ electron gas in a continuum limit.

In a typical antidot lattice, the lattice constant is $a=2000 \AA$ and the electron concentration is $n \sim 2 \times 10^{11}$ $\mathrm{cm}^{-2}$ giving the Fermi wavelength $\lambda_{F} \sim 500 \AA$. Therefore, $a / \lambda_{F}=3.77$ is used in the followings. Further, we choose $\beta=2, d_{f} / a=0.035$, and $l_{e} / a=4$, which are reasonable for realistic systems. ${ }^{2,3}$ It should be noted that $l_{e}$ is longer than $a$.

In the absence of the disorder there are two channels (numbers of one-dimensional subbands below the Fermi level) between nearest-neighbor antidots for lattices with $d / a=0.7$ and there is only a single channel for $d / a=0.8 .^{5}$ In the presence of the diameter fluctuation $d_{f} / a=0.035$ the channel number can fluctuate between 1 and 2 for both of $d / a=0.7$ and 0.8 .

The conductance of finite-size antidot lattices has been calculated through a direct recursive Green's function technique without introduction of S matrices. ${ }^{2,3}$ The present $\mathrm{S}$ matrix formulation reproduces results of such direct calculations quite well, demonstrating the validity of the formulation itself. Such calculations show that the fluctuation in the antidot diameter is not effective in reducing the conductance itself (corresponding to the fact that it essentially acts as a long-range scatterer) but plays important roles in quantum interference effects.

A set of $\mathrm{S}$ matrices for quantum-wire junctions is prepared such that five antidots can take three different values of the diameter, i.e., averaged, larger, and smaller, and three different kinds of impurity configurations are chosen for each antidot-diameter configuration. Each antidot lattice is given by a combination of $\mathrm{S}$ matrices randomly selected among $3^{5} \times 3=729$ kinds of quantum-wire junctions.

\section{B. Finite-size scaling}

The localization length is calculated based on a finitesize scaling method. ${ }^{33,34}$ Using S matrices, we calculate the inverse localization length for infinitely long quasione-dimensional (1D) systems with periodic boundary condition in the direction of width. We define an S matrix $S^{(n)}$ for quasi-1D system with $n$ slits as

$$
\left(\begin{array}{c}
\mathbf{A}_{1} \\
\mathbf{A}_{2}
\end{array}\right)=\left(\begin{array}{ll}
S_{11}^{(n)} & S_{12}^{(n)} \\
S_{21}^{(n)} & S_{22}^{(n)}
\end{array}\right)\left(\begin{array}{l}
\mathbf{B}_{1} \\
\mathbf{B}_{2}
\end{array}\right),
$$

with $\mathbf{A}$ and $\mathbf{B}$ being vectors consisting of amplitudes of out-going waves and in-coming waves, respectively, whose dimension is the number of modes, and the subscripts 1 and 2 indicating the sides of the system. Then, the inverse localization length $\alpha$ for a system with the width of $L$ is given by

$$
\alpha(L)=-\lim _{n \rightarrow \infty} \frac{1}{2 \sqrt{3} n a} \ln \left[\operatorname{Tr}\left(S_{21}^{(n)} S_{21}^{(n) \dagger}\right)\right],
$$

where the length of a slit is chosen as $\sqrt{3} a$ and the trace is taken over elements for traveling modes.

Consider connection of a slit with $\mathrm{S}$ matrix $T$ with an $n$-slit system with $S^{(n)}$ given by Eq. (2.3).

$$
\left(\begin{array}{l}
\mathbf{C}_{1} \\
\mathbf{C}_{2}
\end{array}\right)=\left(\begin{array}{ll}
T_{11} & T_{12} \\
T_{21} & T_{22}
\end{array}\right)\left(\begin{array}{l}
\mathbf{D}_{1} \\
\mathbf{D}_{2}
\end{array}\right),
$$

where elements of $\mathbf{C}$ and $\mathbf{D}$ are amplitudes of out-going and in-coming waves, respectively. Figure 2 shows the schematic illustration of the system. Connecting the side denoted by 2 of the long system with that denoted by 1 of a single slit, that is, imposing $\mathbf{A}_{2}=\mathbf{D}_{1}$ and $\mathbf{B}_{2}=\mathbf{C}_{1}$ on Eqs. (2.3) and (2.5), we get an $\mathrm{S}$ matrix for combined system consisting of $n+1$ slits as

$$
\left(\begin{array}{l}
\mathbf{A}_{1} \\
\mathbf{C}_{2}
\end{array}\right)=\left(\begin{array}{ll}
S_{11}^{(n+1)} & S_{12}^{(n+1)} \\
S_{21}^{(n+1)} & S_{22}^{(n+1)}
\end{array}\right)\left(\begin{array}{l}
\mathbf{B}_{1} \\
\mathbf{D}_{2}
\end{array}\right),
$$

with

$$
\begin{aligned}
& S_{11}^{(n+1)}=S_{11}^{(n)}+S_{12}^{(n)}\left[I-T_{11} S_{22}^{(n)}\right]^{-1} T_{11} S_{21}^{(n)}, \\
& S_{12}^{(n+1)}=S_{12}^{(n)}\left[I-T_{11} S_{22}^{(n)}\right]^{-1} T_{12}, \\
& S_{21}^{(n+1)}=T_{21}\left[I-S_{22}^{(n)} T_{11}\right]^{-1} S_{21}^{(n)}, \\
& S_{22}^{(n+1)}=T_{22}+T_{21}\left[I-S_{22}^{(n)} T_{11}\right]^{-1} S_{22}^{(n)} T_{12},
\end{aligned}
$$


where $I$ is a unit matrix.

Consider the off-diagonal element $S_{21}^{(n+1)}$ which is needed for the calculation of $\alpha(L)$. This is given by the product of $S_{21}^{(n)}$ and a term including $S_{22}^{(n)}$ and becomes exponentially small after a lot of combinations. However, we can always calculate $S_{21}^{(n+1)}$ with the use of the normalized $S_{21}^{(n)}$. Because $S_{22}^{(n+1)}$, which is necessary for $S_{21}^{(n+2)}$ in the next step of calculation, does not depend on $S_{21}^{(n)}$. Therefore, we can perform calculations of $\alpha(L)$ with high accuracy.

We determine the inverse localization length $\alpha_{2 \mathrm{D}}$ of a $2 \mathrm{D}$ system based on the scaling hypothesis ${ }^{33,34}$ that the dimensionless inverse localization length $\alpha L$ is represented by a single scaling function $f\left(\alpha_{2 \mathrm{D}} L\right)$ independent of the parameters such as randomness, i.e., $l_{e}$ and $d_{f}$, and magnetic fields:

$$
\alpha L=f\left(\alpha_{2 \mathrm{D}} L,\{V\}\right) .
$$

We shall leave the possibility of dependence of $f$ on antidot potential parameters $\{V\}$ such as aspect ratio $d / a$, the shape of the model potential, a kind of lattice structure, and so on. Under this scaling hypothesis we reduce each curve of the width dependence of $\alpha$ for various magnetic fields to a single common curve by a shift in the horizontal direction. The amount of the shift is proportional to the localization length in a $2 \mathrm{D}$ system.

The absolute value of $\alpha_{2 D}$ is not determined by this method alone because there remains an ambiguity corresponding to a multiplication of $\alpha_{2 \mathrm{D}}$ by a common scale factor. The common scale factor and therefore the absolute value of $\alpha_{2 \mathrm{D}}$ are determined in such a way that the geometric average of $\alpha_{2}$ of finite-size scaling results near zero magnetic field, where localization is strong, is equal to that of the previous Thouless-number results. ${ }^{5}$

In the case that the critical exponent is determined and very high accuracy is required, possible correction to scaling may have to be considered. ${ }^{35,36}$ In this paper we shall neglect such correction because we are interested in the rough position and qualitative behavior of critical magnetic fields.

In the following finite-size scaling analysis, we perform calculations for systems with width $L / a=8,12$, and 16 at all magnetic fields and in addition those for $L / a=4$ in a strong localization regime and for $L / a=24$ in a weak localization regime. Statistical errors of the numerical results for the localization length of each 1D system are $\lesssim 3 \%$.

\section{NUMERICAL RESULTS}

\section{A. Scaling}

Figures 3 and 4 show the inverse localization length $\alpha$ of quasi-1D systems with $d / a=0.7$ and 0.8 , respectively, as a function of magnetic field $\Phi / \Phi_{0}$ where $\Phi$ is a flux passing through a unit cell. Results for width $L / a=8$, 12 , and 16 are shown. Larger $\alpha$ and stronger dependence on the system size means stronger localization.

In Fig. 3 with $d / a=0.7$ the localization becomes weaker for $\Phi / \Phi_{0} \lesssim 3$ with the increase of the magnetic field and $\alpha$ oscillates around a small value in the range $-0.7 \lesssim \ln (\alpha L) \lesssim-0.1$ for $\Phi / \Phi_{0} \gtrsim 3$. In Fig. 4 with $d / a=0.8$ it is clear that the localization becomes weaker for $\Phi / \Phi_{0} \lesssim 8$ with the increase of the magnetic field and stronger again for $\Phi / \Phi_{0} \gtrsim 8$. This suggests that the localization length takes maximum at $\Phi / \Phi_{0} \sim 8$.

In the case of $d / a=0.8$ the localization is stronger than that in the case of $d / a=0.7$ at the same magnetic field. This is to be expected because in the case of $d / a=0.8$ electrons are strongly confined in the region surrounded by antidots as compared with those in the case of $d / a=0.7$.

Figures 5 and 6 show the same results of $\alpha$ as Figs. 3 and 4 , respectively, as a function of width $L$ for each $\Phi / \Phi_{0}$. In Figs. 5 and 6 data for $L / a=4$ in the strong localization regime and in Fig. 6 those for $L / a=24$ in the weak localization regime are also plotted. In Fig. 5 the system-width dependence for $d / a=0.7$ can be clearly seen though it is unclear in Fig. 3.

Figure 7 shows the results of scaling of the data in Figs. 5 and 6 where the data at integer values of $\Phi / \Phi_{0}$ are plotted for simplicity. In the case of $d / a=0.8$ the data are well scaled into a single common curve and the finite-size scaling works well. In the case of $d / a=0.7$ the finite-size scaling approximately works well, but there is some ambiguity in the weak localization regime, i.e., the region of small $\alpha L$. Because the scaling function depends generally on the universality class, the inverse localization length for zero and nonzero magnetic fields should be analyzed separately. ${ }^{37,35}$

The inset of Fig. 7 shows the result replotted in such a way that the scaling function for $d / a=0.7$ agrees with that for $d / a=0.8$ in the strong localization region by a shift in the horizontal direction. A scaling function seems to depend on the aspect ratio $d / a$.

Figures 8(a) and 9(a) show the resulting inverse localization length $\alpha_{2 \mathrm{D}}$ for $d / a=0.7$ and 0.8 , respectively, as a function of a magnetic field. Figures 8(b) and 9(b) show the blowup of the region near zero magnetic field of $0 \leq \Phi / \Phi_{0} \leq 1$, where filled circles are the results of finitesize scaling and open ones those of the Thouless-number method. ${ }^{5}$

There are three common features in Figs. 8 and 9. One is an oscillation with a period of $\Phi_{0} / 2$ near zero magnetic field, more clearly seen in Figs. 8(b) and 9(b). Another is an oscillation with a period of $\Phi_{0}$ appearing in higher magnetic fields $\Phi / \Phi_{0} \gtrsim 1$ for $d / a=0.7$ and $\Phi / \Phi_{0} \gtrsim 6$ for $d / a=0.8$. The other is a dip structure on which the previous $\Phi_{0}$ oscillations are imposed. The dip is clearly seen at $\Phi / \Phi_{0} \sim 8$ in the case of $d / a=0.8$ as expected from the results of Fig. 6 , while in the case of $d / a=0.7$ the averaged inverse localization length exhibits an unclear minimum at around $\Phi / \Phi_{0} \sim 4$ due to a strong oscillation.

\section{B. Localization oscillations}

Near zero magnetic field, as can be seen in Figs. 8(b) and $9(\mathrm{~b})$, the inverse localization length takes peaks at $\Phi / \Phi_{0}=0$ and $1 / 2$, i.e., the localization becomes strong at these magnetic fields. This is considered to arise from 
the effective change of the universality class, ${ }^{5}$ because an antidot lattice may approximately be regarded as a lattice described by a tight-binding model.

In the presence of a magnetic field, the system has no time reversal symmetry and belongs to a unitary class with $\eta=2$ where $\eta$ is the exponent indicating strength of the level repulsion in a random matrix theory. ${ }^{38} \mathrm{At}$ special magnetic fields of $\Phi / \Phi_{0}=n / 2$ with $n=0,1, \cdots$, however, the Hamiltonian is reduced to a real symmetric matrix and the system has time reversal symmetry and belongs to the orthogonal class with $\eta=1$. In this case the localization becomes stronger.

The oscillations with a period $\Phi_{0}$ are seen in the higher magnetic-field region of $\Phi / \Phi_{0} \gtrsim 1$ for $d / a=0.7$ and $\Phi / \Phi_{0} \gtrsim 6$ for $d / a=0.8$. In both cases the inverse localization length almost always takes a dip at the integer values of $\Phi / \Phi_{0}$.

It is known that an $\mathrm{AB}$ type oscillation of the resistivity appears superimposed on the commensurability peak determined by the condition $2 R_{c}=a$ (corresponding to $\Phi / \Phi_{0} \sim 6.5$ in the present case). ${ }^{6,7}$ This AB type oscillation originates from periodic orbits encircling an antidot and its phase varies sensitively as a function of various parameters such as energy, confinement potentials, etc. ${ }^{7-10}$

Therefore, the $\Phi_{0}$ oscillation of the inverse localization length is not related to semiclassical periodic orbits but to a periodic change of the band structure similar to the so-called Hofstadter butterfly spectrum. ${ }^{39}$ In fac$t$, the butterfly spectrum always takes the largest band width when the flux passing through a plaquette is an integer multiple of the flux quantum $\Phi_{0}$. The large band width gives rise to a largest effective group velocity and therefore to a weaker localization effect.

In higher magnetic fields $\Phi / \Phi_{0} \gtrsim 10$ for $d / a=0.7$, the period becomes slightly smaller than $\Phi_{0}$. It may be attributed to the fact that the band structure is modified by the strong magnetic field itself and the oscillation may deviate from the exact $\Phi_{0}$ period.

\section{Critical magnetic field}

In the case of $d / a=0.8$ shown in Fig. $9(\mathrm{a})$, the inverse localization length takes a minimum at $\Phi / \Phi_{0} \sim 8$. The estimated localization length exceeds 5000 in units of $a$ which is about $1 \mathrm{~mm}$ for typical lattice constant $a=2000$ $\AA$, which is macroscopically large. Although it is quite difficult to draw a definite conclusion whether states are really extended or not, we can practically regard states to be extended at this magnetic field and the corresponding field as an insulator-quantum Hall transition point where the temperature dependence of the resistivity vanishes.

In the case of $d / a=0.7$ in Fig. 8(a), it is more difficult to determine such insulator-quantum Hall transition point because localization is weak in a wide magnetic field range due to weak confinement and the $\Phi_{0}$ oscillation with the large amplitude. When this oscillation is averaged over, the inverse localization length seems to have a minimum at around $\Phi / \Phi_{0} \sim 4$.

In the following we shall consider the case $d / a=0.8$ in more detail. Figure 10 shows the magnetic field dependence of $\alpha$ for $L / a=8,12$, and 16 at energies (a)
$E / E_{F}=1$, (b) 0.75 , (c) 0.625 , and (d) 0.5. Similar calculations for $d / a=0.7$ requires much computation time because of the weaker localization effect and are left for a future study.

Figure 10(a) is the same as Fig. 4 and therefore, the magnetic field $\Phi / \Phi_{0} \sim 8$ is an insulator-quantum Hall transition point. In the case $E / E_{F}=0.75$ in Fig. $10(\mathrm{~b}), \alpha$ is nearly independent of the system size at about the same magnetic field $\Phi / \Phi_{0} \sim 8$. In addition to it, there seems to be another higher critical magnetic field at $\Phi / \Phi_{0} \sim 33$ where $\alpha$ takes a local minimum and does not depend on the system size approximately. It is confirmed that there are no other magnetic fields between $8 \lesssim \Phi / \Phi_{0} \lesssim 33$ where $\alpha$ does not depend on the system size. At $E / E_{F}=0.625$ in Fig. $10(\mathrm{c})$, the lower critical field stays almost same while higher one becomes smaller to be $\Phi / \Phi_{0} \sim 18$. At $E / E_{F}=0.5$ in Fig. $10(\mathrm{~d})$, there is no critical magnetic field where $\alpha$ does not depend on the system size.

Figure 11 shows the energy spectrum for $d / a=0.8$ as a function of the magnetic field. The lowest three Landau levels $(N=0,1$, and 2$)$ in the absence of the antidot potential are plotted by solid lines. It can be seen that energy bands converge to the Landau levels in the high magnetic field region. As the magnetic field is decreased, the energy bands deviate from the Landau levels toward the higher energy region and are broadened due to the existence of the antidot potential.

Open inverted triangles indicate the critical magnetic fields obtained in Fig. 10. The states at higher critical magnetic fields of $\Phi / \Phi_{0} \sim 33$ at $E / E_{F}=0.75$ and $\Phi / \Phi_{0} \sim$ 18 at $E / E_{F}=0.625$ are obviously associated with the extended states in the center of the lowest Landau level because they seem to belong to the energy bands which converge to the lowest Landau level in sufficiently high magnetic fields.

The dotted line in Fig. 11 shows a schematic illustration of the behavior of extended states obtained by an appropriate interpolation. In sufficiently high magnetic fields, an extended state lie near the center of the lowest Landau level. As the magnetic field becomes weak, its energy decreases but lies at a higher energy than the Landau-level center. At a critical field $\Phi / \Phi_{0} \sim 8$ it rapidly goes up in energy. No extended states associated with higher Landau levels are present below the Fermi energy. This is presumably because the higher Landau levels are more strongly affected by the antidot potential and their extended states are raised higher than the Fermi level.

Mixing between the Landau levels is essential for the insulator-quantum Hall transition. Therefore, a critical magnetic field is qualitatively expected to become larger for larger $d / a$ because of a larger confinement and a stronger disturbance of Landau levels. Therefore, the smaller critical field $\Phi / \Phi_{0} \sim 4$ in Fig. 8(a) for $d / a=0.7$ than for $d / a=0.8$ is reasonable.

\section{DISCUSSION}

Our numerical results for $d / a=0.7$ can qualitatively explain recent experimental results of insulator-quantum Hall transition. ${ }^{1}$ In experiments, the resistivity near zero magnetic field oscillates with a period $\Phi_{0} / 2$ for about two periods, i.e., $0 \lesssim \Phi / \Phi_{0} \lesssim 1$. The oscillation was analyzed 
based on the variable range hopping and ascribed to the oscillation of the localization length. The numerical results show that the amplitude of the $\Phi_{0} / 2$ oscillation is $\Delta \alpha / \alpha_{0} \sim 0.3$ for $d / a=0.7$ where $\alpha_{0}$ is the localization length at zero magnetic field (the results of the finitesize scaling for $\Phi / \Phi_{0}=0$ and $1 / 2$ being ignored). This is in reasonable agreement with the experimental results $\Delta \alpha / \alpha_{0} \sim 0.2 .^{1}$

With the increase of the magnetic field, the $\Phi_{0} / 2$ oscillation disappears and a $\Phi_{0}$ oscillation appears in experiments. The behavior of this change in the period from $\Phi_{0} / 2$ to $\Phi_{0}$ is qualitatively in agreement with the numerical results for $d / a=0.7$. When the conductivity oscillates with the period $\Phi_{0}$, it takes peaks almost exactly at integer values of $\Phi / \Phi_{0}$ in the experiments. This is consistent with the numerical result that the localization length has a peak when $\Phi / \Phi_{0}$ is an integer. Further, the insulator-quantum Hall transition point of the calculated results for $d / a=0.7$ is $\Phi / \Phi_{0} \sim 4$ in good agreement with the experimental results of $\Phi / \Phi_{0} \sim 3.9$.

In experiments a wide Hall plateau for the filling factor $\nu=2$ was observed but no plateau for higher filling factors, showing that extended states associated with the higher Landau levels never cross the Fermi energy. This is consistent with the behavior of extended states shown in Fig. 11 for $d / a=0.8$, although the parameters are different.

Our results are considered to be general in the case of a sufficiently large aspect ratio $d / a$ but may become inapplicable in systems with small $d / a$. In fact, the presence of two distinct oscillations of the localization length with periods $\Phi_{0} / 2$ and $\Phi_{0}$ is likely to be due to the strong confinement in antidot lattices with large $d / a$ and may be obscured and disappear with the decrease of $d / a$. Such study for systems with smaller $d / a$ is left as a future issue.

\section{SUMMARY}

In a summary, we have numerically studied localization in antidot lattices subjected to a perpendicular magnetic field. Two different kinds of oscillations of the localization length have been obtained. One is that with a period of $\Phi_{0} / 2$ near zero magnetic field and the other that with a period of $\Phi_{0}$ in higher magnetic fields. A critical magnetic field corresponding to an insulatorquantum Hall transition point has been obtained also. At a critical magnetic field the extended states are suddenly raised in energy and disappear in weaker fields. The results are in good agreement with recent experiments.

\section{ACKNOWLEDGMENTS}

This work is supported in part by Grant-in-Aid for COE Research from the Ministry of Education, Culture, Sports, Science, and Technology (12CE2004 "Control of Electrons by Quantum Dot Structures and Its Application to Advanced Electronics"). Some numerical calculations were performed on HITACHI H9000V L2000 in
Supercomputer Center, Institute for Solid State Physics, University of Tokyo. One of the authors (S.U.) was supported by JSPS.

*Present address: RIKEN, 2-1 Hirosawa, Wako, Saitama 351-0198, Japan

\section{References}

1 F. Nihey, M. A. Kastner, and K. Nakamura, Phys. Rev. B 55, 4085 (1997).

2 T. Nakanishi and T. Ando, Phys. Rev. B 54, 8021 (1996).

3 T. Nakanishi and T. Ando, Physica B 227, 127 (1996).

4 B. L. Al'tshuler, A. G. Aronov, and B. Z. Spivak, Pis'ma Zh. Eksp. Teor. Fiz. 33, 101 (1981) [JETP Lett. 33, 94 (1981)].

5 S. Uryu and T. Ando, Phys. Rev. B 58, 10583 (1998).

6 F. Nihey and K. Nakamura, Physica B 184, 398 (1993)

7 D. Weiss, K. Richter, A. Menschig, R. Bergmann, H. Schweizer, K. von Klitzing, and G. Weimann, Phys. Rev. Lett. 70, 4118 (1993)

8 S. Ishizaka, F. Nihey, K. Nakamura, J. Sone, and T. Ando, Phys. Rev. B 51, 9881 (1995)

9 S. Uryu and T. Ando, Phys. Rev. B 53, 13613 (1996)

10 S. Uryu and T. Ando, Physica B 256-258, 388 (1998).

11 T. Ando, J. Phys. Soc. Jpn. 52, 1740 (1983).

12 H. Aoki and T. Ando, Phys. Rev. Lett. 54, 831 (1985).

13 T. Ando and H. Aoki, J. Phys. Soc. Jpn. 54, 2238 (1985).

14 E. Abrahams, P. W. Anderson, D. C. Licciardello, and T. V. Ramakrishnan, Phys. Rev. Lett. 42, 673 (1979).

15 D. E. Khmel'nitzkii, Pis'ma Zh. Eksp. Teor. Fiz. 38, 454 (1983) [JETP Lett. 38, 552 (1983)].

16 R. B. Laughlin, Phys. Rev. Lett. 52, 2304 (1984).

17 S. Kivelson, D. H. Lee, and S. C. Zhang, Phys. Rev. B 46, 2223 (1992).

18 H. W. Jiang, C. E. Johnson, K. L. Wang, and S. T. Hannahas, Phys. Rev. Lett. 71, 1439 (1993).

19 T. Wang, K. P. Clark, G. F. Spencer, A. M. Mack, and W. P. Kirk, Phys. Rev. Lett. 72, 709 (1994).

20 S. V. Kravchenko, W. Mason, J. E. Furneaux, and V. M. Pudalov, Phys. Rev. Lett. 75, 910 (1995).

21 T. Ando, J. Phys. Soc. Jpn. 53, 3126 (1984).

22 T. Ando, J. Phys. Soc. Jpn. 55, 3199 (1986).

23 T. Ando, Phys. Rev. B 40, 5325 (1989).

24 D. Z. Liu, X. C. Xie, and Q. Niu, Phys. Rev. Lett. 76, 975 (1996).

25 X. C. Xie, D. Z. Liu, B. S. Sundaram, and Q. Niu, Phys. Rev. B 54, 4966 (1996).

26 K. Yang and R. N. Bhatt, Phys. Rev. Lett. 76, 1316 (1996).

27 D. N. Sheng and Z. Y. Weng, Phys. Rev. Lett. 78, 318 (1997).

28 Y. Hatsugai, K. Ishibashi, and Y. Morita, Phys. Rev. Lett. 83, 2246 (1999).

29 D. J. Thouless, M. Kohmoto, M. P. Nightingale, and M. den Nijs, Phys. Rev. Lett. 49, 405 (1982).

30 D. P. Arovas, R. N. Bhatt, F. D. M. Haldane, P. B. Littlewood, and R. Ranmel, Phys. Rev. Lett. 60, 619 (1988).

31 Th. Koschny, H. Potempa, and L. Schweitzer, Phys. Rev. Lett. 86, 3863 (2001).

32 D. N. Sheng, Z. Y. Weng, and X. G. Wen, condmat/00031117.

33 A. MacKinnon and B. Kramer, Phys. Rev. Lett. 47, 1546 (1981).

34 A. MacKinnon and B. Kramer, Z. Phys. B 53, 1 (1983).

35 K. Slevin and T. Ohtsuki, Phys. Rev. Lett. 78, 4083 
(1997).

36 B. Huckestein, Phys. Rev. Lett. 72, 1080 (1994).

37 K. B. Efetov, Adv. Phys. 32, 53 (1983).

38 F. J. Dyson, J. Math. Phys. 3, 140 (1962).

39 D. Hofstadter, Phys. Rev. B 14, 2239 (1976)

\section{Figure Captions}

Fig. 1. Schematic illustrations of (a) quantum-wire junction array as a model of hexagonal antidot lattice and (b) a single quantum-wire junction used in calculations of $\mathrm{S}$ matrix.

Fig. 2. Schematic illustration of connecting a long system with $n$ slits to a system with a single slit.

Fig. 3. Magnetic-field dependence of the inverse localization $\alpha$ for $L / a=8,12$, and 16 in the case of $d / a=0.7$.

Fig. 4. Magnetic-field dependence of $\alpha$ for $L / a=8,12$, and 16 in the case of $d / a=0.8$.

Fig. 5. System-size dependence of $\alpha$ for various magnetic fields in the case of $d / a=0.7$.

Fig. 6. System-size dependence of $\alpha$ for various magnetic fields in the case of $d / a=0.8$.

Fig. 7. Scaling of the data for $d / a=0.7$ and 0.8 which are shown in Figs. 5 and 6, respectively. The inset shows the same scaling functions except that the scaling plot for $d / a=0.7$ is horizontally shifted in such a way that the data in a strong localization region agree with those for $d / a=0.8$.

Fig. 8. Calculated inverse localization length $\alpha_{2 \mathrm{D}}$ for $d / a=0.7$ as a function of magnetic field $\Phi / \Phi_{0}$. In (b) a blowup of the region around a zero magnetic field of $0 \leq \Phi / \Phi_{0} \leq 1$ is shown.

Fig. 9. Calculated inverse localization length $\alpha_{2 \mathrm{D}}$ for $d / a=0.8$ as a function of $\Phi / \Phi_{0}$. A blowup of the region around a zero magnetic field of $0 \leq \Phi / \Phi_{0} \leq 1$ is shown in (b).

Fig. 10. Magnetic-field dependence of $\alpha$ for $d / a=0.8$ and $L / a=8,12$, and 16 at (a) $E / E_{F}=1$, (b) 0.75 , (c) 0.625 , and (d) 0.5 .

Fig. 11. Energy spectrum for $d / a=0.8$ as a function of $\Phi / \Phi_{0}$. Solid lines indicate the lowest three Landau levels in the absence of the antidot potential. The inverted triangles show the critical magnetic fields obtained from the results in Fig. 10. The dotted line shows the expected behavior of the energy of extended states as a function of the magnetic field. 
(a)

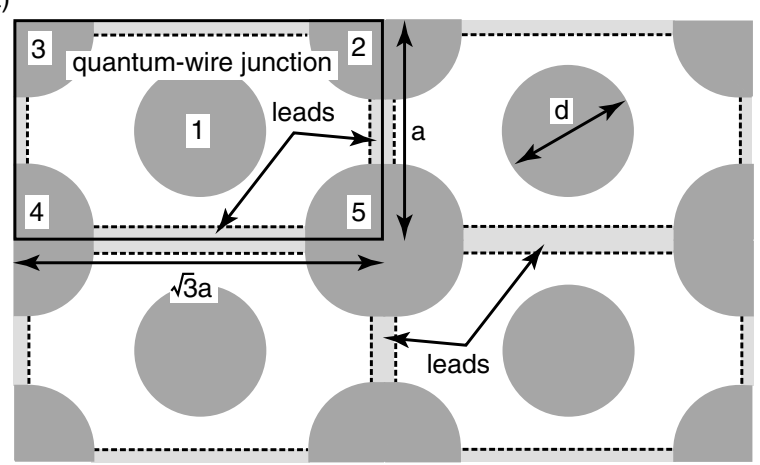

(b)

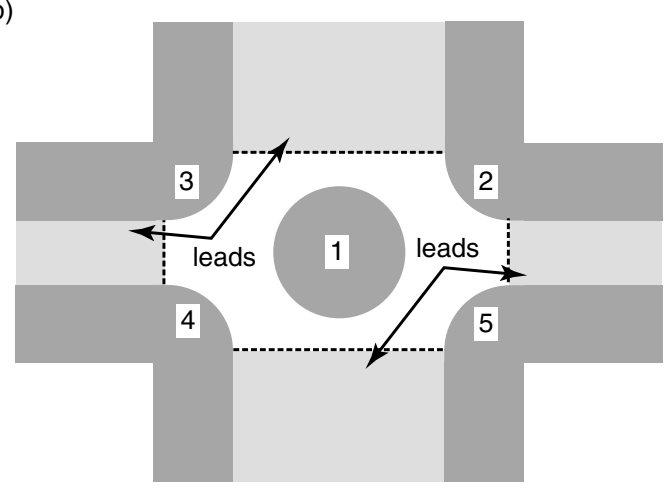

Fig. 1

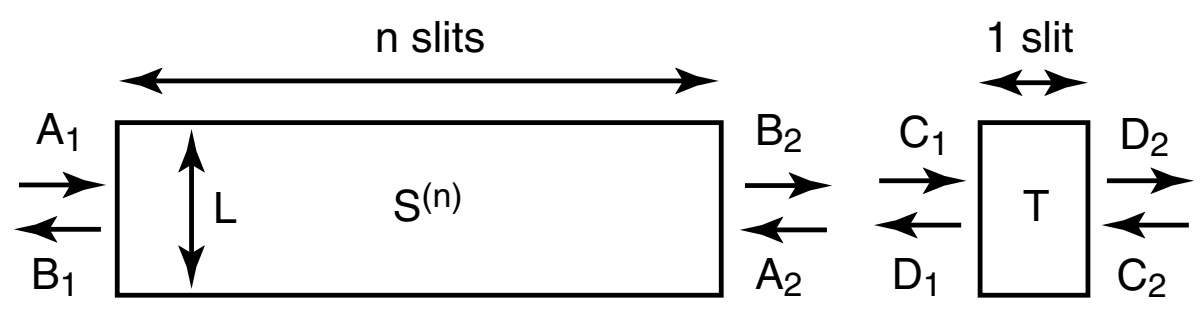

Fig. 2

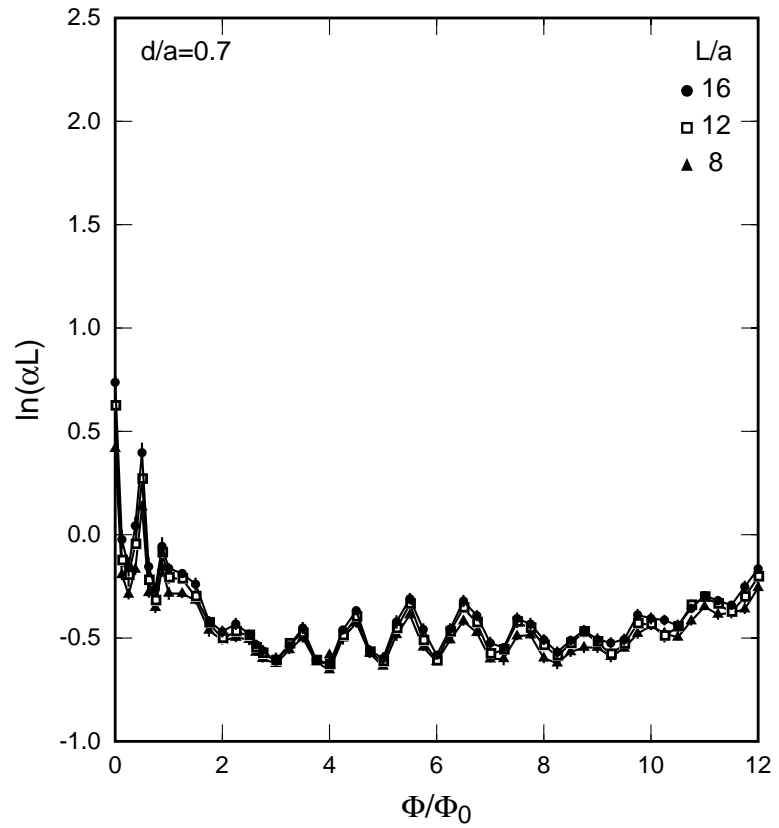

Fig. 3

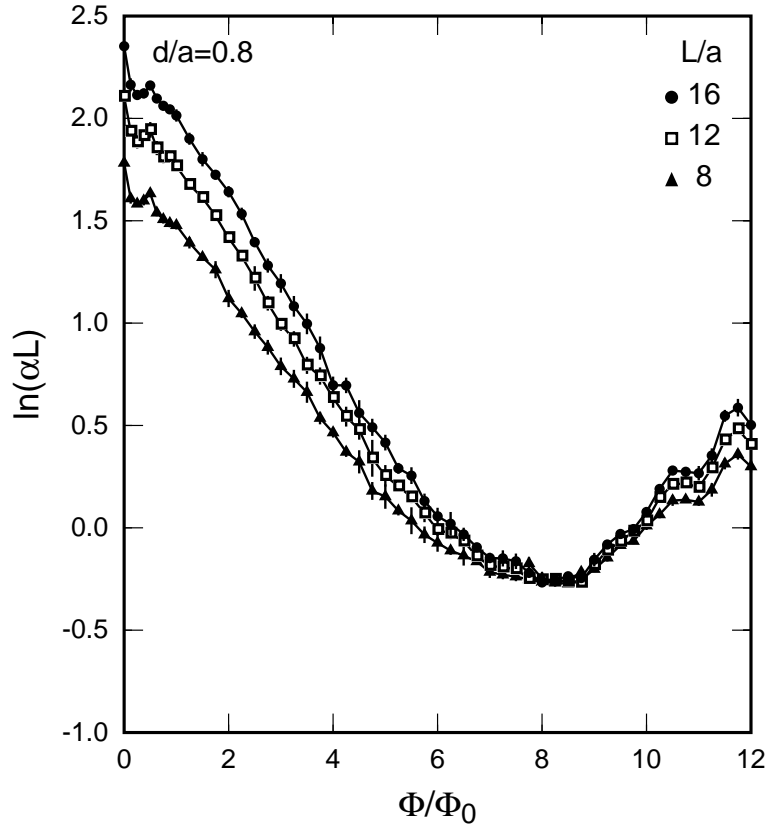

Fig. 4 

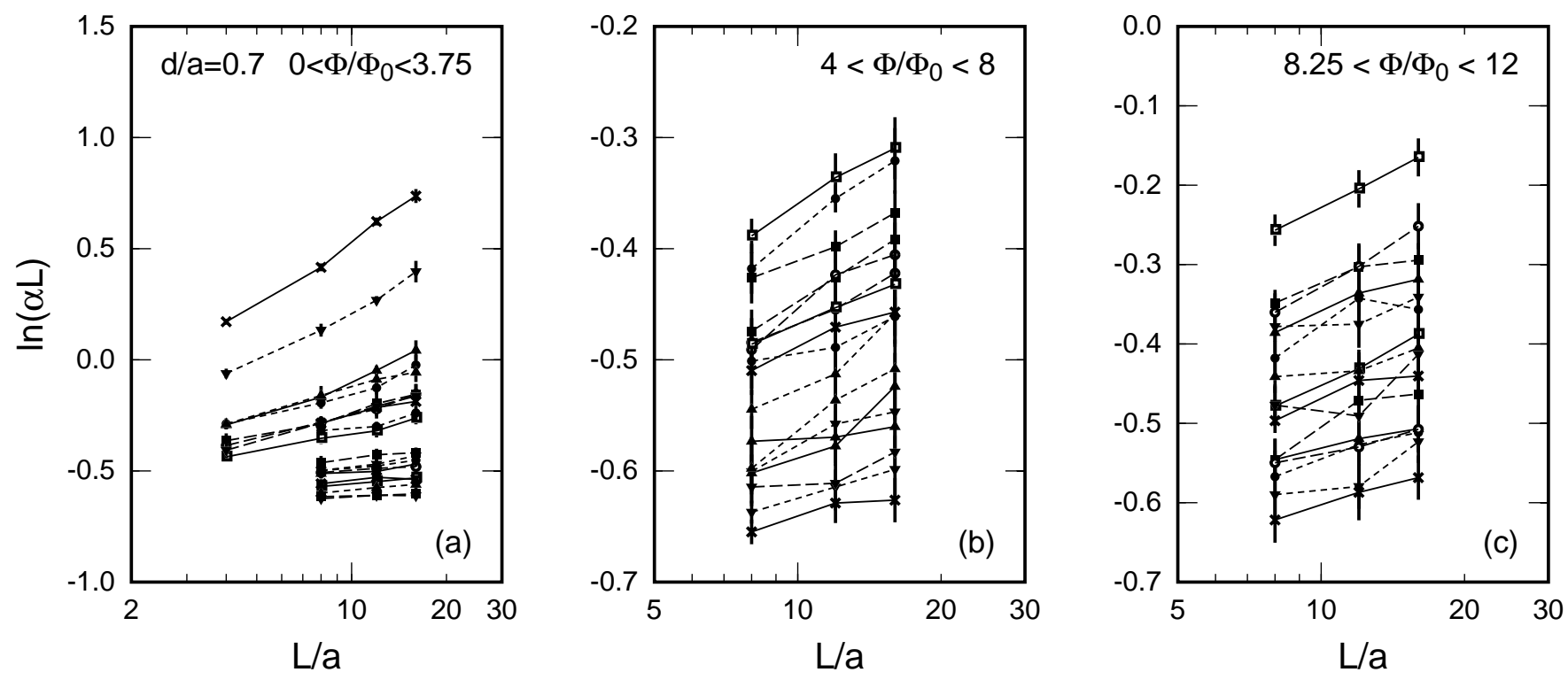

Fig. 5
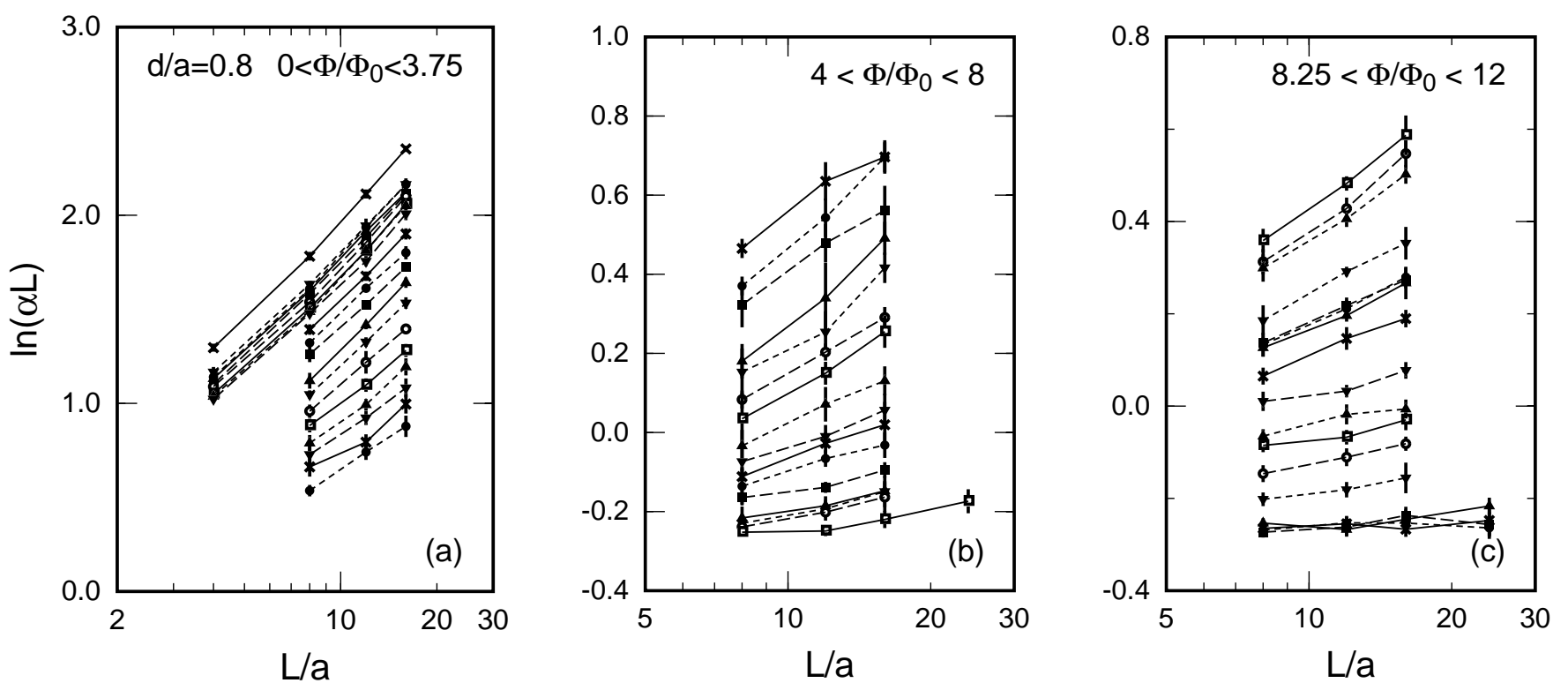

Fig. 6 


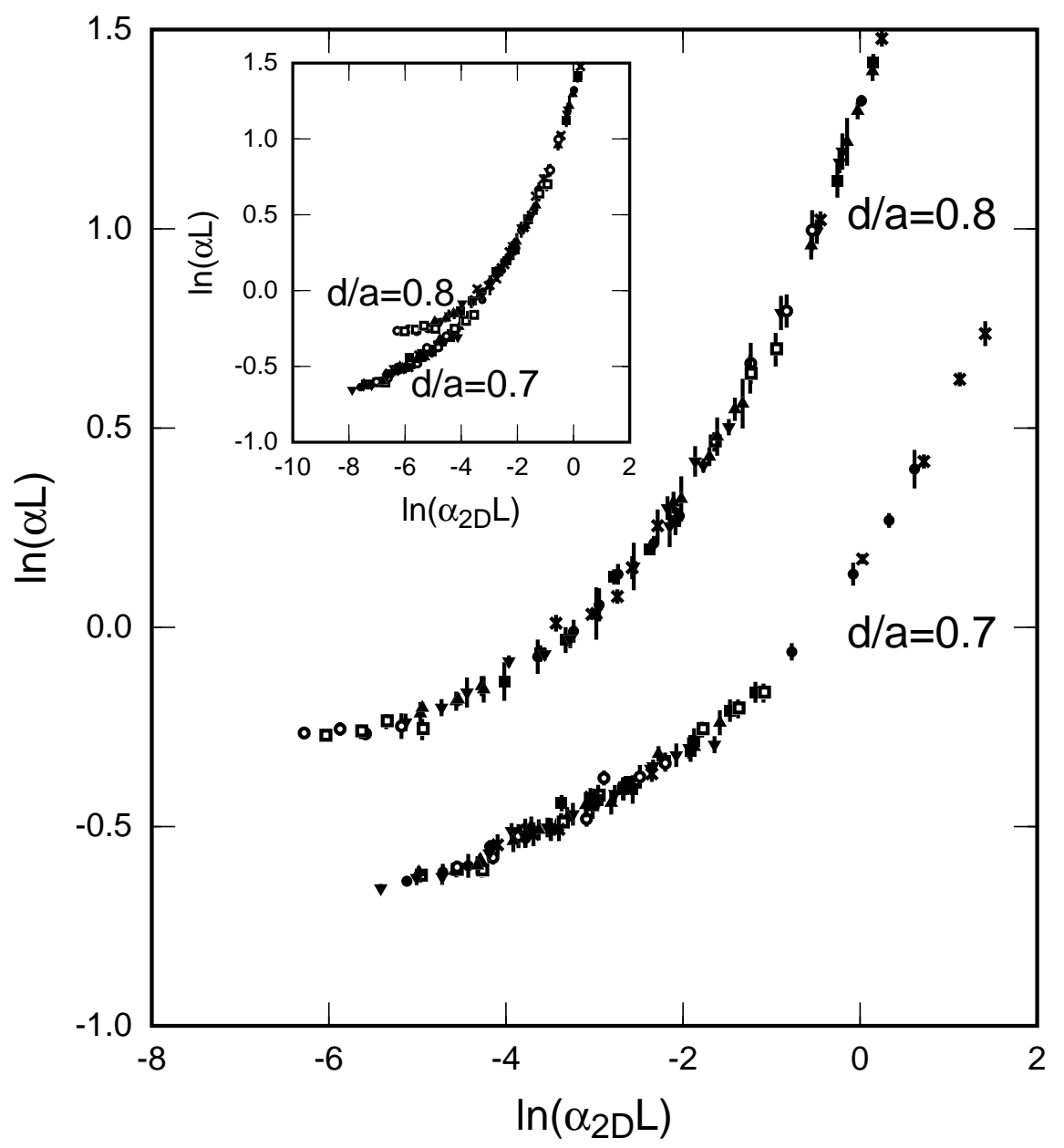

Fig. 7 


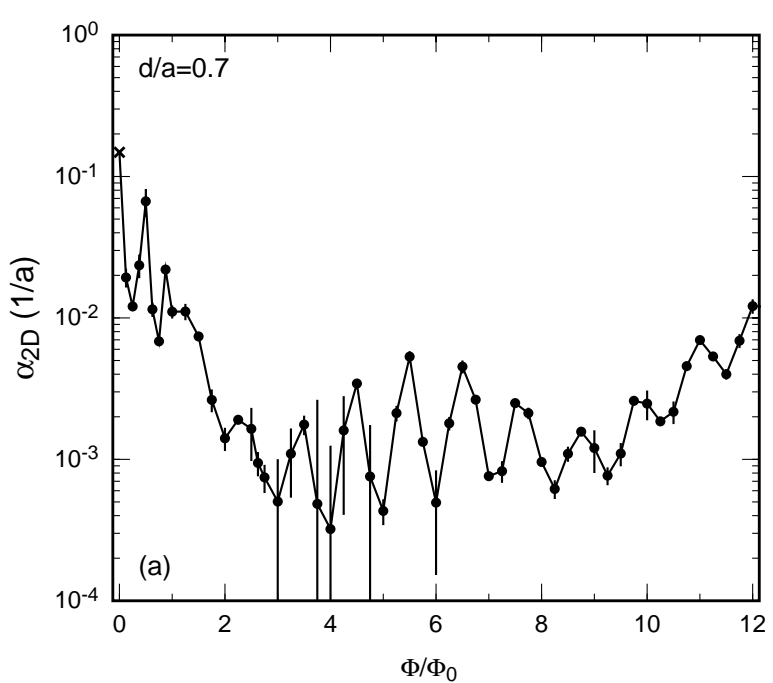

Fig. 8(a)

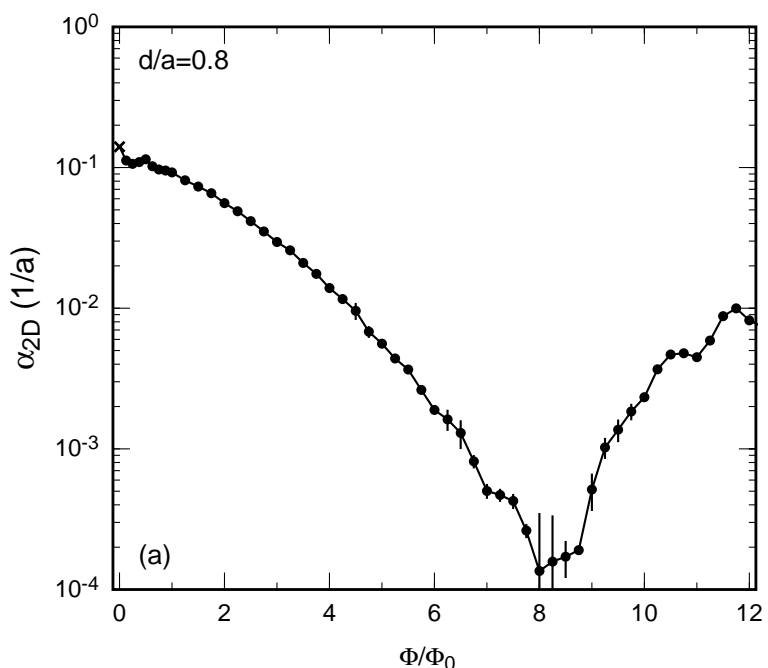

Fig. 9(a)

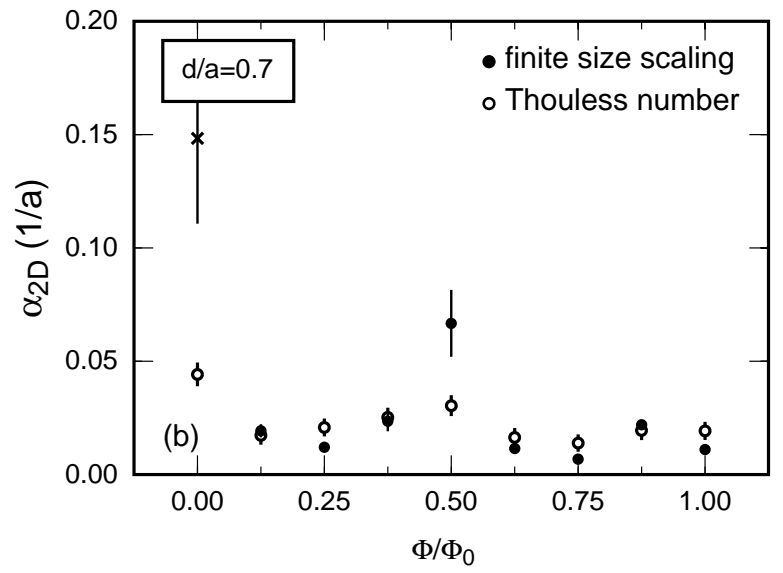

Fig. 8(b)

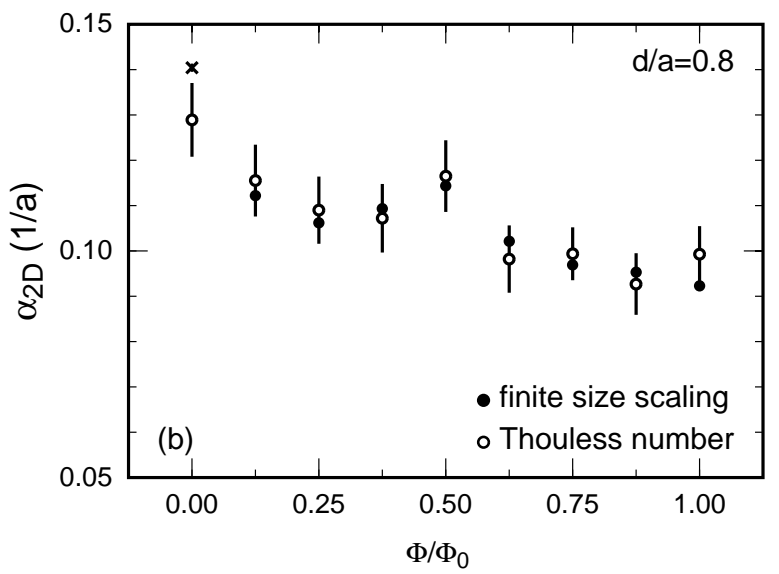

Fig. 9(b) 

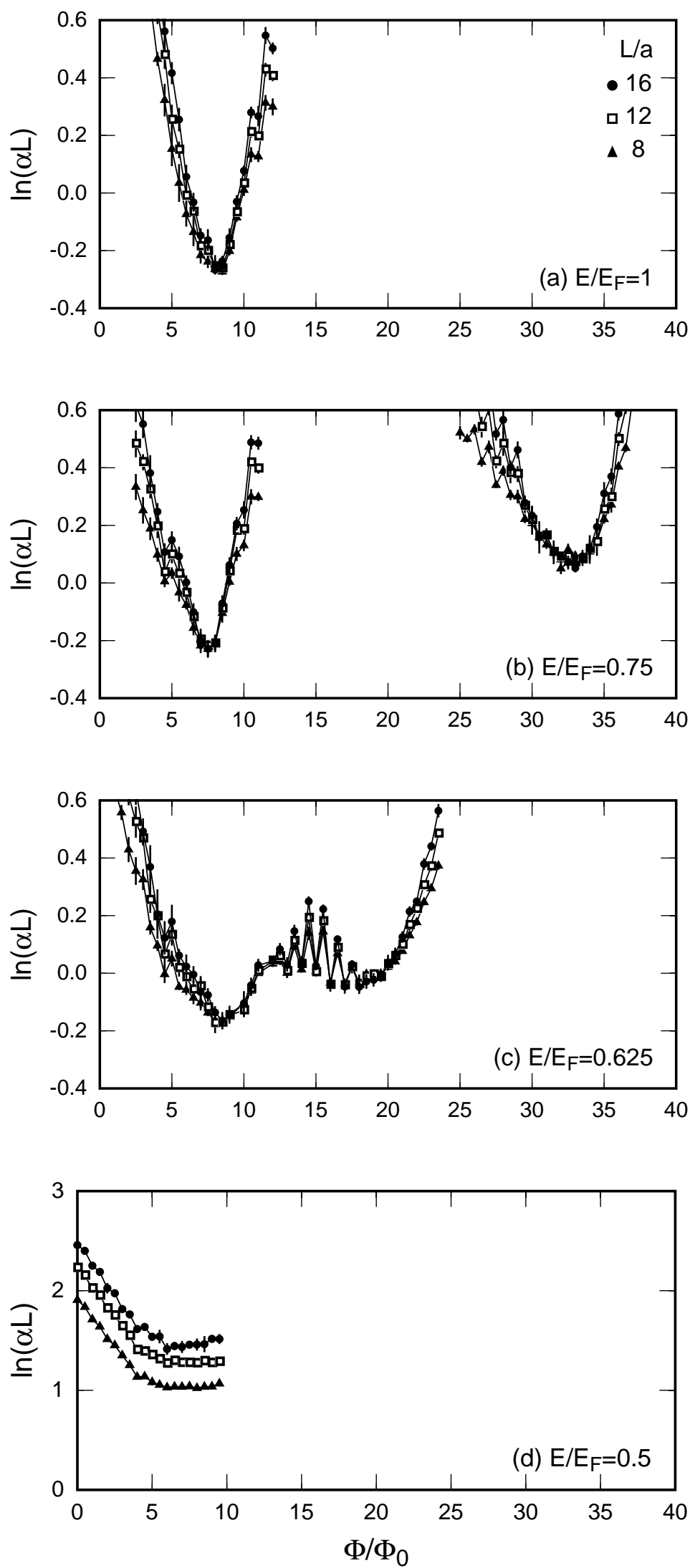

Fig. 10 


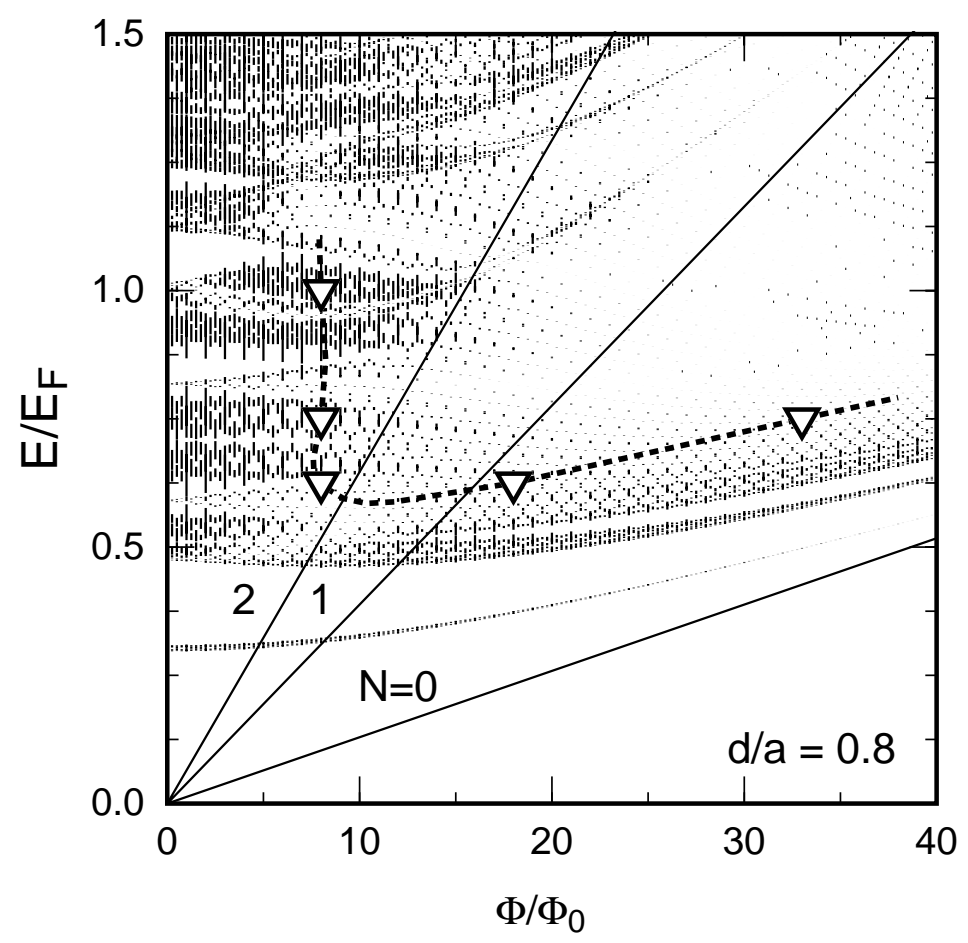

Fig. 11 